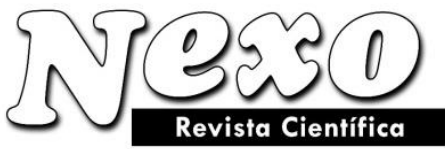

ISSN-E 1995-9516

Universidad Nacional de Ingeniería COPYRIGHT @ (UNI). TODOS LOS DERECHOS RESERVADOS http://revistas.uni.edu.ni/index.php/Nexo https://doi.org/10.5377/nexo.v34i06.13125

Vol. 36, No. 06, pp. 1649-1657/Diciembre 2021

(c) (i) $(-$

\title{
Fibra de coco como aditivo de mejoramiento en la elaboración de bloques
}

\section{Coconut fiber as improvement additive in the preparation of blocks}

\author{
Mauricio Alejandro Rivera Miranda ${ }^{1}$, Lorenzo Antonio Jarquín Flores ${ }^{1}$, Alejandro Armando \\ Obando Francis ${ }^{1}$, Julio Cesar Araúz Urbina ${ }^{1}$, Juan Asdrúbal Flores-Pacheco ${ }^{1,2 *}$ \\ 1 Bluefields Indian \& Caribbean University - BICU. Escuela de Ingeniera Civil, apartado postal 88, \\ Avenida Universitaria, Bluefields, Nicaragua. \\ 2 Bluefields Indian \& Caribbean University - BICU. Dirección de Investigación y Postgrado (DIP), \\ apartado postal 88, Avenida Universitaria, Bluefields, Nicaragua. \\ * Correspondencia: asdrubal.flores@do.bicu.edu.ni / ORCID https://orcid.org/0000-0001-6553-7202
}

(recibido/received: 13-septiembre-2021; aceptado/accepted: 05-diciembre-2021)

\section{RESUMEN}

La incorporación de la fibra de coco en los bloques elaborados en la ciudad de Bluefields, brindo información relevante durante la evaluación de sus propiedades físicas y mecánicas, esto a través de la interpretación de los datos obtenidos de los ensayos de comprensión que se hicieron en el Laboratorio Julio-Adolfo de la Universidad Centro Americana (UCA). Dichos bloques se analizaron en los diferentes días de fraguado que son de 7 y 28 días respectivamente analizando por cada ensayo 9 bloques, 6 bloques de 6 ", ( 3 con el $5 \%$ de aditivo y 3 con el $20 \%$ de aditivo) además 6 bloques de 4 " ( 3 con el $5 \%$ de aditivo y 3 con el $20 \%$ de aditivo. Los resultados de los ensayos se determinaron que los bloques tenían evidencian que la implementación de las fibras permite al concreto la adherencia de los materiales permitiendo al concreto presente agrietamiento normal después de la falla, pero es controlado por las fibras quienes impiden la prolongación de dichas grietas haciendo que estas terminen allí, brindando ductilidad.

Palabras claves: Esfuerzo de rotura, mampostería, mezcla de mortero.

\begin{abstract}
The incorporation of coconut fiber in the blocks elaborated in the city of Bluefields, provided relevant information during the evaluation of its physical and mechanical properties, through the interpretation of the data obtained from the compression tests carried out at the Julio-Adolfo Laboratory of the Central American University (UCA). These blocks were analyzed in the different setting days, which are 7 and 28 days respectively, analyzing 9 blocks for each test, 6 blocks of 6" (3 with 5\% of additive and 3 with 20\% of additive) and 6 blocks of 4" (3 with 5\% of additive and 3 with $20 \%$ of additive. The results of the tests determined that the blocks had evidence that the implementation of the fibers allows the concrete the adherence of the materials allowing the concrete to present normal cracking after the failure, but it is controlled by the fibers that prevent the prolongation of these cracks making them end there, providing ductility.
\end{abstract}

Keywords: Breaking stress, masonry, mortar mix 


\section{INTRODUCCIÓN}

Las viviendas que existen en Nicaragua se han realizado en su mayoría con materiales de construcción no idóneos o no certificados, debido a que gran parte de la población nicaragüense son de escasos recursos económicos o habitan en comunidades alejadas donde es difícil el acceso a materiales de construcción que sean de buena calidad y estén certificados, esto genera que dichas viviendas no logren cumplir con los términos técnicos de construcción que describe los reglamentos y manuales de construcción tanto nacionales como internacionales, esto provoca que dichas estructuras puedan estar en riesgo

En la ciudad de Bluefields generalmente, las infraestructuras urbanas verticales se construyen con mampostería confinada utilizando bloques no certificados, ocasionando que están sean construcciones vulnerables. Esto se debe a que en la ciudad no se cuentan con empresas bloqueras certificadas, aunque en la región hay proveedores de materiales de construcción certificados pero su elevado costo económico, hace que pocos pobladores puedan adquirirlos, siendo para resto de población la única opción de construir con materiales de baja calidad. También la carencia de laboratorios de materiales de construcción en la ciudad no permite conocer la calidad de los bloques que se fabrican, así también las dosificaciones empleadas por las empresas locales no son mediante los estándares adecuados de la NTON 12008-16.

Por eso fue importante analizar la fibra de coco como aditivo en la fabricación de bloques de cemento, arena, material cero y agua, para establecer si este es apto usarlo en la construcción. La determinación de las propiedades y características de los bloques con fibras de coco como aditivo, se realizó por medio de los análisis de ensayos de laboratorios, en los laboratorios de la UCA, así también se compararán los resultados con la NTON 12-008-16.

\section{MATERIALES Y MÉTODOS}

\subsection{Localización del estudio}

Este estudio se realizó en la ciudad de Bluefields cabecera municipal del municipio del mismo nombre. Es un estudio experimental del tipo descriptivo porque se hizo una descripción detallada del comportamiento específico del bloque en los que se utilizó el aditivo de fibra en las diferentes pruebas de laboratorio, con enfoque cuantitativo por que se utilizaron datos numéricos para determinar la factibilidad del aditivo de las fibras de coco en los bloques, y de corte transversal porque se hizo en un lapso de tiempo.

\subsection{Universo - Población}

La población de referencia de este estudio está constituida por tres bloqueras legalmente establecidas y registradas ante la Alcaldía Municipal de Bluefields. El numero de bloques a elaborar se toma en base a la producción media por hora en cada sitio. Dicho esto, la muestra es no probabilística al no contar con suficientes elementos para su estimación.

\subsection{Muestra y muestreo}

Tabla 1. Cantidad de bloques de 6" y 4" analizados.

\begin{tabular}{ccccc}
\hline $\begin{array}{r}\text { Tipo de } \\
\text { bloques }\end{array}$ & Cantidad de bloques & $\begin{array}{c}\text { Porcentaje (\%) de fibra de } \\
\text { coco }\end{array}$ & Días de curado & Proporción \\
\hline $6 "$ & 3 & 5 & 7 & $1: 5: 4$ \\
& 3 & 20 & 28 &
\end{tabular}




\begin{tabular}{ccccc}
\hline $\begin{array}{c}\text { Tipo de } \\
\text { bloques }\end{array}$ & Cantidad de bloques & $\begin{array}{c}\text { Porcentaje (\%) de fibra de } \\
\text { coco }\end{array}$ & Días de curado & Proporción \\
\hline & 3 & 5 & 7 & \\
3 & 20 & 28 & \\
3 & 5 & 28 & $1: 5: 3$ \\
3 & Sin fibra & 28 & \\
\hline 3 & 5 & 7 & $1: 5: 4$ \\
3 & 20 & 28 & \\
3 & 5 & 28 & $1: 5: 3$ \\
\hline 3 & 20 & 28 & \\
\hline
\end{tabular}

Como muestra se elaboraron 36 bloques de los cuales 18 fueron de 6" y los otros 18 de 4". En la tabla se puede apreciar cómo fue la distribución de los bloques en cuanto a su tamaño, porcentaje de fibra de coco, días de curado y proporción.

\subsection{Técnicas e instrumentos de recolección de datos}

\subsubsection{Cálculo de la dosificación}

El cálculo de la dosificación se realizó en base a la proporción que ocupa las boqueras de la ciudad la cual es 1:5:4 (una de cemento, cinco de arena, cuatro de material cero). A esta se le realizo cálculos de volumen con el fin de ver cuántos kilogramos de material se iba a ocupar, valores que están reflejadas en la tabla 2.

Tabla 2. Cantidad de bloques según el porcentaje de fibra de coco.

\begin{tabular}{cccccccc}
\hline $\begin{array}{c}\text { Tipo de } \\
\text { bloques }\end{array}$ & $\begin{array}{c}\text { Cantidad de } \\
\text { bloques }\end{array}$ & $\begin{array}{c}\text { Porcentaje } \\
\text { de fibra de coco }\end{array}$ & $\begin{array}{c}\text { Cemento } \\
(\mathbf{k g})\end{array}$ & $\begin{array}{c}\text { Arena } \\
(\mathbf{k g})\end{array}$ & $\begin{array}{c}\text { Material } \\
\text { cero }(\mathbf{k g})\end{array}$ & $\begin{array}{c}\text { Fibra de } \\
\text { coco }(\mathbf{k g})\end{array}$ & $\begin{array}{c}\text { Agua } \\
(\mathbf{L t s})\end{array}$ \\
\hline \multirow{2}{*}{ 6" } & 6 & 20 & 10.625 & 53.125 & 42.5 & 0.57 & 3.75 \\
& 6 & 5 & 10.625 & 53.125 & 42.5 & 0.37 & 3.75 \\
\hline \multirow{2}{*}{$4 "$} & 6 & 20 & 6.84 & 34.08 & 27.33 & 0.53 & 2.42 \\
& 6 & 20 & 6.84 & 34.08 & 27.33 & 0.34 & 2.42 \\
\hline
\end{tabular}

\subsubsection{Elaboración de los bloques}

Para la elaboración de los bloques se utilizó mortero de cemento en dosificaciones 1:5:4 (una de cemento, cinco de arena, cuatro de material cero) y 1:5:3 (una de cemento, cinco de arena, tres de material cero), añadiéndole en su mayoría fibra de coco de $5 \mathrm{~cm}$ de largos en proporciones de $5 \%$ y $20 \%$ (5\% solo a la proporción 1:5:3) del volumen de la mezcla, dejándolo en reposo en siete días algunos y otros en 28 días. Las dimensiones de estos bloques estas descritas en la tabla 1 respetando lo que indica el reglamento de la NTON 12 006-8.

Tabla 3. Dimensiones de los bloques de 6" y 4".

\begin{tabular}{ccccc}
\hline \multirow{2}{*}{ Bloques } & \multicolumn{3}{c}{ Dimensión cm } & \multirow{2}{*}{ Área Neta mm $\mathbf{~ m m}^{\mathbf{2}}$} \\
\cline { 2 - 4 } & Largo cm & Ancho cm & Alto cm & \\
\hline Bloques 6" & 39 & 15 & 19 & 41200 \\
Bloques 4" & 39 & 10 & 19 & 24600 \\
\hline
\end{tabular}

\subsubsection{Ensayos de laboratorios}

Los ensayos de laboratorios se realizaron en el laboratorio Julio Adolfo de la Universidad Centro Americana (UCA), ubicado en la ciudad de Managua. Primeramente, se realizaron ensayos físicos 
mecánicos de la materia prima que se utilizaron en la elaboración de los bloques siendo la absorción a la fibra de coco. En los bloques fue el contenido de humedad, resistencia a la comprensión, densidad, absorción los ensayos realizados. También se realizaron ensayos de muestreo de agregados y análisis granulométrico a los agregados.

\section{RESULTADOS Y DISCUSIÓN}

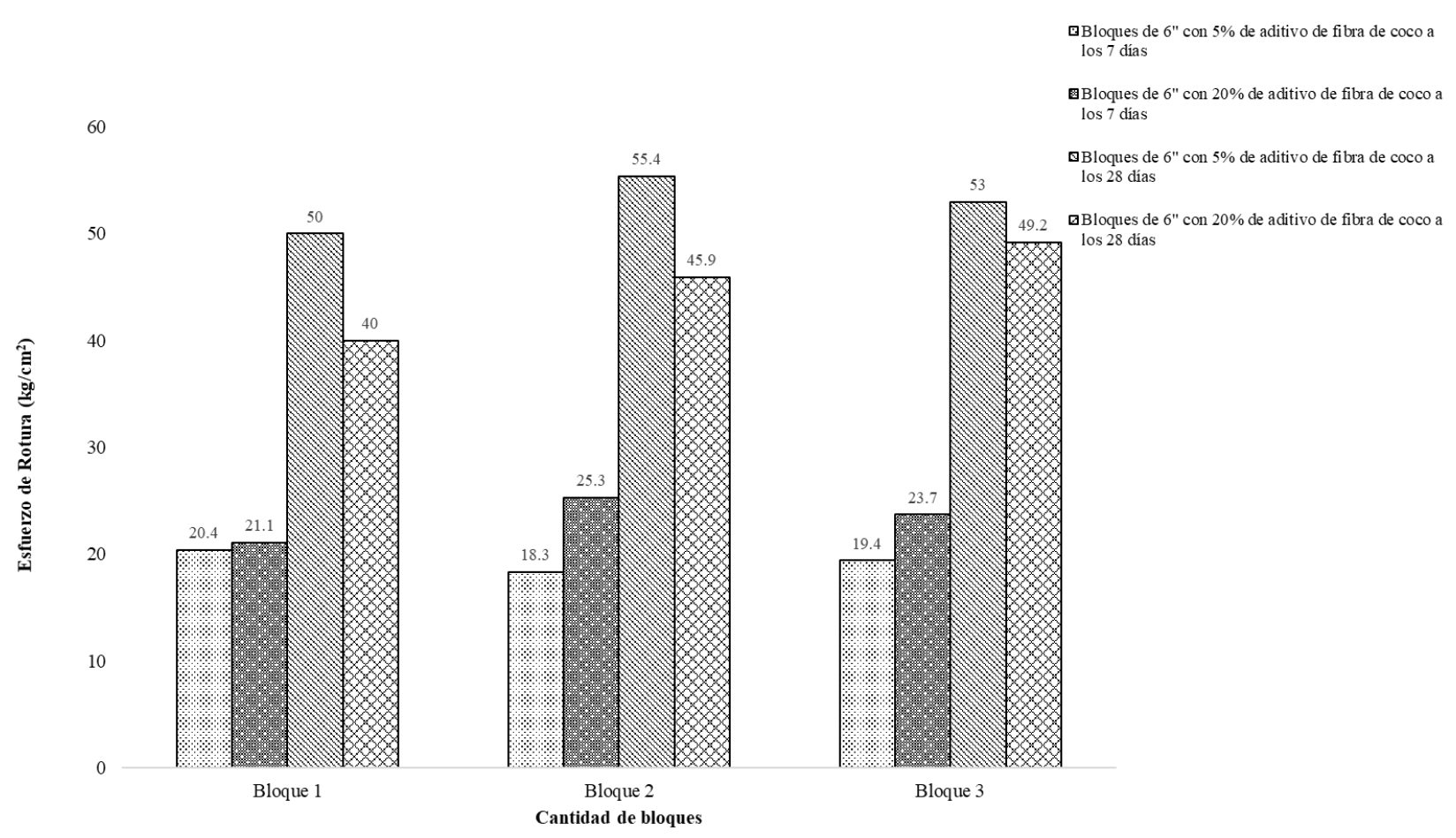

Figura 1. Comparación de bloques de 6" con aditivos de fibra de coco a los 5\% y $20 \%$.

En la figura 1 se representa los resultados de los esfuerzos de rotura en $\mathrm{kg} / \mathrm{cm}^{2}$ de los bloques de 6" obtenidos de los ensayos de laboratorios a comprensión, el cual para su fabricación se ocupó una dosificación de 1:5:4 (una de cemento, cinco de arena, cuatro de material cero) dosificación que utilizan las boqueras de la ciudad. En dicho ensayo se utilizaron un total 12 bloques de los cuales 6 estaban con un tiempo de reposo a 7 días y el resto a 28 días. De los 6 bloques, tanto los que estaban en reposo a los 7 días como a los que estaban a los 28 días, se analizaron 3 que tuvieran como aditivo un $5 \%$ de fibra de coco y los otros 3 con un $20 \%$, dando como resultados que el bloque que tenía $5 \%$ de fibra de coco como aditivo y con un tiempo de reposo de 28 días, tuviera los mejores resultados en cuanto a la resistencia a la comprensión, promediando un esfuerzo de rotura aproximadamente de $52.8 \mathrm{~kg} / \mathrm{cm}^{2}$, en cambio el que tuvo menor promedio de esfuerzo de rotura fue el bloque que tenía $5 \%$ de fibra de coco pero con un tiempo de reposo de 7 días siendo el valor de $19.36 \mathrm{~kg} / \mathrm{cm}^{2}$ semejante al promedio que presentaron los bloques a $20 \%$ con el mismo tiempo de curado el cual fue de $23.36 \mathrm{~kg} / \mathrm{cm}^{2}$. Se debe destacar que ninguno de estos bloques cumplió con los $120 \mathrm{~kg} / \mathrm{cm}^{2}$ que establece la norma de NTON 12 008-16. 


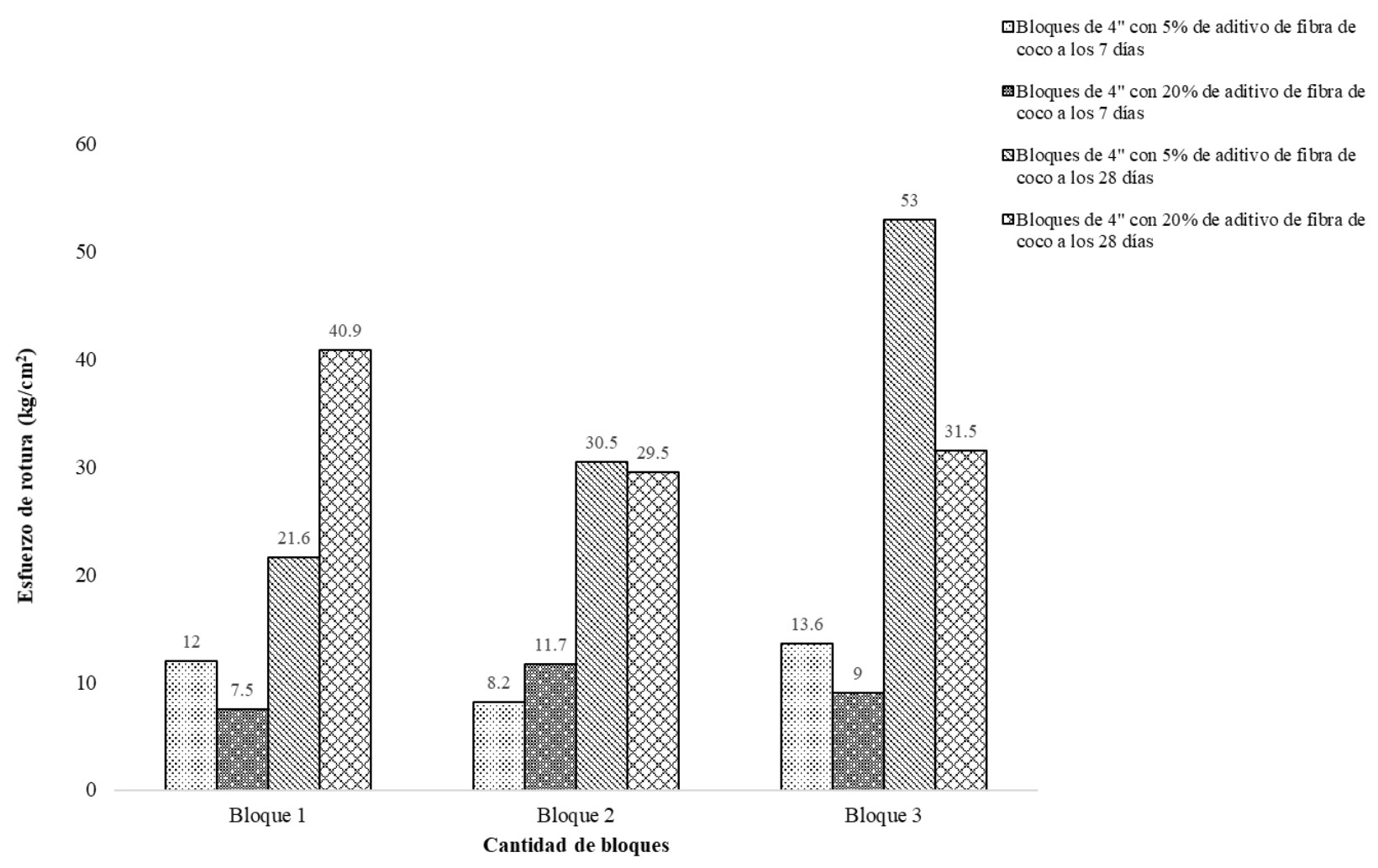

Figura 2. Comparación de bloques de 4" con aditivos de fibra de coco a los 5\% y $20 \%$.

Para el análisis de laboratorios de bloques de 4" se hizo el mismo procedimiento en cuanto a la dosificación, cantidad de bloques, los tiempos de reposo y la cantidad de porcentaje de fibras de coco, teniendo que el mejor promedio de esfuerzo de rotura, según los ensayos de laboratorios a comprensión, el bloque con $5 \%$ de fibra de coco con tiempo de reposo a los 28 días, teniendo un valor como promedio de $35.03 \mathrm{~kg} / \mathrm{cm}^{2}$, valor que es parecido al que se obtuvo en los bloques con $20 \%$ de fibra de coco con el mismo tiempo de reposo el cual es de $33.96 \mathrm{~kg} / \mathrm{cm}^{2}$, el bloque que se obtuvo menor valor fueron los bloques que tenían como aditivo de fibra de coco un $20 \%$ y un tiempo de reposo de 7 días siendo de 9.4 $\mathrm{kg} / \mathrm{cm}^{2}$. Estos promedios están por debajo con respecto a los presentado en los bloques de 6", pudiendo tener como influencia el volumen de los bloques a la hora de hacer los ensayos. Tampoco ninguno de estos bloques cumplió con los $120 \mathrm{~kg} / \mathrm{cm}^{2}$ que establece la norma de NTON 12 008-16. 
๑Bloques de $6 "$ con $5 \%$ de aditivo fibra de coco

国 Bloques de $6 "$ con $20 \%$ de aditivo fibra de coco

¿Bloques de 6" sin aditivo ferreteria Sevilla

\begin{tabular}{|c|c|c|}
\hline & 120 & घBloques de $6 "$ sin aditivo ferretería \\
\hline 120 & 37 & $\begin{array}{l}\text { Gonzalez } \\
\text { aBloques de 6" sin aditivo ferretería Quinto }\end{array}$ \\
\hline 100 & & $\begin{array}{l}\text { (Resistencia promedio minimo para } 3 \text { piezas } \\
\text { NTON } 12 \text { 008-16 }\end{array}$ \\
\hline
\end{tabular}

0

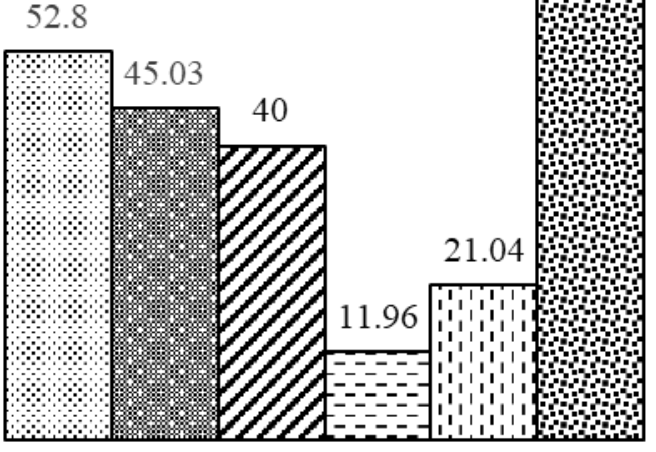

Figura 3. Comparación de la resistencia a comprensión promedio de los bloques con aditivo y bloque sin aditivos elaborados en la ciudad de Bluefields con la dosificación 1:5:4.

En la figura 3 se muestra que los bloques con fibra de coco obtuvieron mejores resultados que los que se hacen de manera artesanal y se comercializan en la ciudad, teniendo el mejor promedio de esfuerzo de rotura el bloque con $5 \%$ de aditivo de fibra de coco obteniendo un resultado de $52.8 \mathrm{~kg} / \mathrm{cm}^{2}$. Sin embargo todos los bloques analizados no cumplen con lo normado en la NTON 12-008-16, la cual indica que para tres pruebas de resistencia de bloques debe de dar como mínimo $120 \mathrm{~kg} / \mathrm{cm}^{2}$ de esfuerzo de rotura, esto puede ser por la dosificación 1:5:4 que se implementó en los bloques analizados, no cumpliendo con la dosificación propuesta por el Instituto Americano de Concreto (ACI en sus siglas en ingles) y el Ministerio de Transporte e Infraestructura (MTI) del país que indican que debe ser una proporción de 1:5:2 elaborando con ella 28 bloques de 6" por bolsa de cemento contrario a la dosificación implementada la cual se pueden elaborar 36 bloques de 6" por bolsa de cemento. También se observó que en el ensayo de laboratorio los bloques que contenían fibras de coco como aditivos no se desintegraban a como lo hacían los bloques artesanales, destacando que las fibras de coco aumentaban la adherencia de los materiales en el bloque, así mismo que los refuerzos de fibra mejoran de varias maneras la tenacidad de la matriz, ya que una grieta que se mueva a través de la matriz encuentra una fibra. Al iniciarse una grieta en la mezcla, fibras aun no rotas puede formar un puente sobre la grieta, lo cual proporciona un esfuerzo compresivo que evita que la grieta se abra, aumentando de esta manera la resistencia de los bloques. 
120

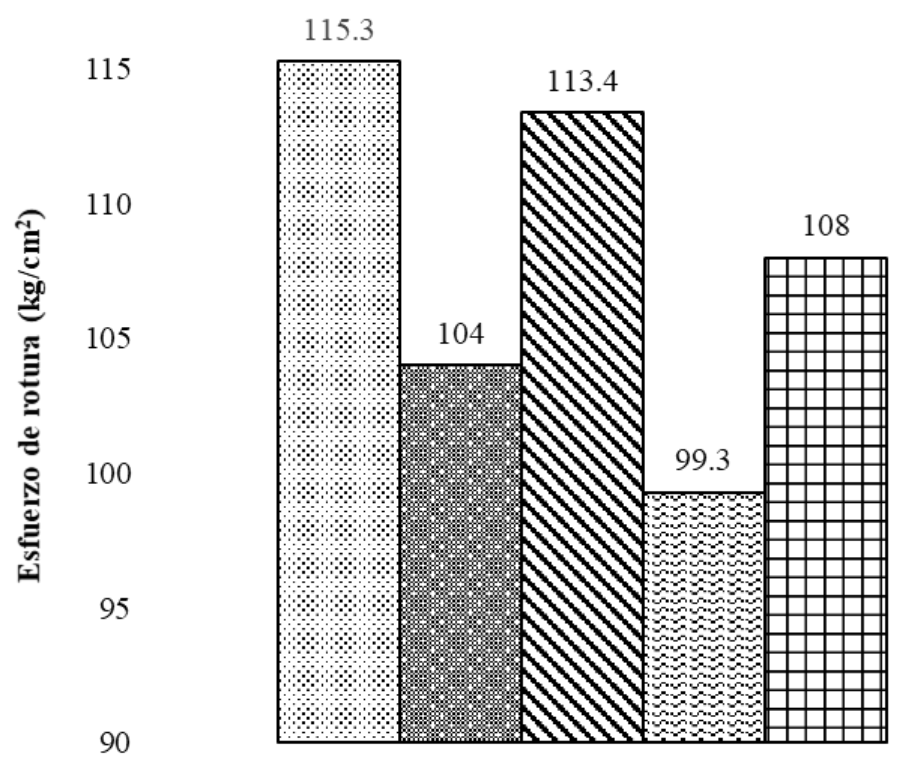

B Bloques de 6" con 5\% de aditivo de fíbra de coco

图Bloques de 6" sin aditivo

$\mathbf{\Delta B l o q u e s}$ de $4 "$ con $5 \%$ de aditivo de fibra de coco

Bloques de $4 "$ sin aditivo

巴Resistencia mínima a la comprensión para una pieza individual NTON 12 008-16

Figura 4. Comparación de la resistencia a comprensión promedio de los bloques con aditivo y sin aditivo aplicando la dosificación propuesta 1:5:3

Se puede observar en la figura 4 que los bloques de 6" y 4" con el 5\% de aditivo de fibra de coco y con un curado a los 28 días, cumplieron con lo que establece la NTON 12 008-16, además que tuvieron mejores resultados que los bloques que no tenían aditivo, estos no cumplieron con la norma. Los bloques de 4" con aditivo fueron el 7.96\% más resistentes que los bloques de 4" sin aditivos, en cambio los bloques de 6" con aditivo fueron $13.652 \%$ más resistentes que los bloques 6" sin aditivo. Se debe destacar que estos bloques se realizaron con una dosificación 1:5:3, la cual fue propuesta de los investigadores. Solo se analizó los bloques al 5\% de aditivo y con un curado de 28 días debido a que los estudios anteriores establecían que los bloques tenían mejor resistencia con menos porcentaje de fibras de coco en su contenido y con 28 días de curado.

Tabla 3. Costo de material para la elaboración de 720 bloques de 4" o la elaboración de 640 bloques de 6" aplicando la dosificación propuesta de 1:5:3 y el aditivo al 5\% de fibra de coco

\begin{tabular}{|c|c|c|c|c|}
\hline Descripción & $\mathbf{U} / \mathbf{M}$ & Cantidad & Costo Unitario & Costo Total \\
\hline Arena & $\mathrm{ft}^{3}$ & 100 & $\$ 1.35$ & $\$ 135.45$ \\
\hline Material Cero & $\mathrm{ft}^{3}$ & 60 & $\$ 1.40$ & $\$ 83.92$ \\
\hline Cemento & $\mathrm{u} / \mathrm{m}$ & 20 & $\$ 10.22$ & $\$ 204.44$ \\
\hline Fibra de coco & $\mathrm{Kg}$ & 28.53 & $\$ 0.51$ & $\$ 14.46$ \\
\hline
\end{tabular}

En la tabla 3 se puede visualizar que al utilizar una dosificación propuesta de 1:5:3 se pueden elaborar 720 bloques de 4" o 640 bloques de 6", gastando aproximadamente $\$ 438.26$ (Cuatrocientos treinta y ocho dólares con veintiséis centavos de dólar). Esto genera que por cada bloque de 4" se tendría que gastar \$0.61 (sesenta y un centavos de dólar) en materiales, a esto se le tendría que sumar el costo de la mano de 
obra que actualmente anda en un promedio de $\$ 0.05$ (cinco centavos de dólar), teniendo un costo total del bloque en su elaboración de $\$ 0.66$ (sesenta y seis centavos de dólar). En cambio, el precio del bloque de 6 " en materiales sería de $\$ 0.68$ (sesenta y ocho centavos de dólar), en mano de obra de $\$ 0.05$ (cinco centavos de dólar), teniendo un gasto para su elaboración de $\$ 0.73$ (setenta y tres centavos de dólar). Sin embargo, agregando los costos indirectos, gasto de administración, utilidades e impuesto en los costos de los bloques de 4" y 6" estos tendrían un costo de \$ 0.87 (ochenta y siete centavos de colar) y \$ 0.97 (noventa y siete centavos de dólar) respectivamente, esto se puede apreciar en los datos que tiene la tabla 4 y 5 .

Tabla 4. Costo de total agregando los costos indirectos de un bloque 4" aplicando la dosificación propuesta de 1:5:3 y el aditivo al $5 \%$ de fibra de coco

\begin{tabular}{|c|c|c|c|c|c|}
\hline ITEM & Descripción & $\mathbf{U} / \mathbf{M}$ & Cantidad & $\begin{array}{c}\text { Costo } \\
\text { Unitario }\end{array}$ & Costo Total \\
\hline 1 & $\begin{array}{l}\text { Fabricación de bloque por } \\
\text { día }\end{array}$ & Unidad & 1 & $\$ 0.66$ & $\$ 0.66$ \\
\hline A & a. Total Costo Directo & & & & $\$ 0.66$ \\
\hline B & $\begin{array}{l}\text { b. Total costo indirecto } \\
(2 \% \text { sobre a) }\end{array}$ & & & & $\$ 0.01$ \\
\hline $\mathrm{C}$ & $\begin{array}{l}\text { c. Administración ( } 9 \% \\
\text { sobre } a+b)\end{array}$ & & & & $\$ 0.0603$ \\
\hline $\mathrm{D}$ & $\begin{array}{l}\text { d. Utilidades ( } 3 \% \text { sobre } \\
a+b+c)\end{array}$ & & & & $\$ 0.022$ \\
\hline E & e. Sub-Total $(a+b+c+d)$ & & & & $\$ 0.7523$ \\
\hline $\mathrm{F}$ & f. I.V.A. (15\% sobre e) & & & & $\$ 0.1128$ \\
\hline \multirow[t]{2}{*}{ G } & $\begin{array}{l}\text { Impuesto municipal } 1 \% \\
\text { sobre }\end{array}$ & & & & $\$ 0.0075$ \\
\hline & $\begin{array}{l}\text { Precio total de la oferta } \\
(\mathrm{e}+\mathrm{f}+\mathrm{g})\end{array}$ & & & & $\$ 0.8726$ \\
\hline
\end{tabular}

Tabla 5. Costo de total agregando los costos indirectos de un bloque 6" aplicando la dosificación propuesta de 1:5:3 y el aditivo al $5 \%$ de fibra de coco

\begin{tabular}{|c|c|c|c|c|c|}
\hline ITEM & Descripción & $\mathbf{U} / \mathbf{M}$ & Cantidad & $\begin{array}{c}\text { Costo } \\
\text { Unitario }\end{array}$ & Costo Total \\
\hline 1 & $\begin{array}{l}\text { Fabricación de bloque por } \\
\text { día }\end{array}$ & Unidad & 1 & $\$ 0.73$ & $\$ 0.73$ \\
\hline A & a. Total Costo Directo & & & & $\$ 0.73$ \\
\hline B & $\begin{array}{l}\text { b. Total costo indirecto } \\
(2 \% \text { sobre a) }\end{array}$ & & & & $\$ 0.01$ \\
\hline $\mathrm{C}$ & $\begin{array}{l}\text { c. Administración ( } 9 \% \\
\text { sobre } a+b)\end{array}$ & & & & $\$ 0.07$ \\
\hline D & $\begin{array}{l}\text { d. Utilidades (3\% sobre } \\
a+b+c)\end{array}$ & & & & $\$ 0.02$ \\
\hline E & e. Sub-Total $(a+b+c+d)$ & & & & $\$ 0.84$ \\
\hline $\mathrm{F}$ & f. I.V.A. (15\% sobre e) & & & & $\$ 0.13$ \\
\hline \multirow[t]{2}{*}{ G } & $\begin{array}{l}\text { Impuesto municipal } 1 \% \\
\text { sobre }\end{array}$ & & & & $\$ 0.01$ \\
\hline & $\begin{array}{l}\text { Precio total de la oferta } \\
(\mathrm{e}+\mathrm{f}+\mathrm{g})\end{array}$ & & & & $\$ 0.97$ \\
\hline
\end{tabular}




\section{CONCLUSIONES}

Se observo que los bloques con fibras de coco no se desintegraban a la hora de hacer los ensayos de laboratorios a comprensión debido a las fibras, haciendo que las fallas fueran graduales, todo lo contrario, con los bloques que no tenían fibras ya que estos se fracturaron en su totalidad. También los resultados obtenidos de ensayos de laboratorios se deducen que a menor porcentaje de fibras de coco que tenga los bloques, mayores serán las resistencias obtenidas debido a la consistencia entre el cemento y las fibras.

Los bloques que se incluyó la fibra de coco a 5\% y $20 \%$ como aditivo y se realizaron con la dosificación que ocupan la empresa bloquearas 1:5:4 no cumplieron con los valores mínimos de la NTON 12 008-16 pero la resistencia a comprensión de los bloques con fibra de coco tuvo un aumento de aproximadamente del $15 \%$ en su esfuerzo de rotura comparado con los bloques que se realizan artesanalmente en la ciudad. La dosificación propuesta de 1:5:3 correspondiente a cemento, arena y material cero, respectivamente para la elaboración de bloques de 6" y 4", y con la inclusión del 5\% de fibra de coco se observó que cumplen con la norma NTON 12 008-16 en ambos casos.

Los precios promedio de los bloques de 6" y 4" que incluyen como aditivo fibras de coco serán de \$1.07 (un dólar con siete centavos de dólar) y \$ 0.98 (noventa y ocho centavos de dólar) respectivamente. Se debe mencionar que estos precios fueron calculados con los costos de los materiales de venta local de la ciudad.

\section{REFERENCIAS}

Brinsa. (2018). BRINSA. Obtenido de https://www.obrinsa.com.ni/index.php/productos-y-servicios/84bloquesde-concreto

Calderón, L. A. (2000). Estudio de materiales cementicios fibrorreforzados y su posible uso como elementos de reparación en estructuras afectadas por corrosión en medio ambiente marino. Santiago del Valle: Universidad del Valle.

Comsión Nacional de Normalización Técnica.(2009). Norma Técnica Obligatoria Nicaraguense NTON 12 008-09. Managua.

Gomez Palacios, S. (2009). Diseño, Experimentacion y Evaluación dle Sistema Constructivo SAM (Sistema de bloque fibrorreforzados con fibra del desecho del fruto del coco, para la construcción de vivienda. Guatemala.

González G., J. F. (15 de Mayo de 2018). Bloques de concreto muy sanos. Obtenido de http://www.imcyc.com/ct2008/oct08/sustentabilidad.htm

González, L. O., \& Quintero G., S. L. (2004). Evaluación del uso de la fibra de la estopa de coco, para mejoramiento de las propiedades mecánicas del concreto. Madrid: ANIFER.

Instituto Costarricense del Cemento y del Concreto . (2007). Manual de construcción con bloques de concreto. San José. 